\title{
Electron Transfer Pathways in Cell
}

Yan Liu

School of Chemical Engineering, Qinghai University, Xining 810016 P R

China

E-mail: liuyan_qhu@163.com

Abstract: Analysis of the electron salvation process data indicates that the electron transfer between the electron donor and acceptor is hindered by the electron salvation process. It is proposed that the electron transfer in the cell environment must be assisted by intermediate messenger called the "transport protein".

Introduction

Electron transfer in the bio-maromolecules, such as the protein and DNA were described as the electron transfer directly from donor to acceptor. However, by analyzed the electron salvation process in water, we set-up a theory on how the electron transfer in endocellular environment.

Theory

Electron transfer in the protein and DNA as well as in the biological systems have received extensive studies in the past decades ${ }^{[1-2]}$. However, the endocellular electron transfer pathways are still not well identified at the molecular level. In the conventional picture, the electron transport was considered as free transport of electron directly from the electron donor to the electron acceptor. Since the endocellular 
environment consists $80 \%$ of water content, which is a strong polar medium, endocellular electron transport can not be free between the donor and the acceptor especially separated by a long distance. On the other hand, the electron has to be solvated and the hydrated electron (e-aq) is transported ${ }^{[3]}$. Experimental data showed that the hydrated electron solvation time was $250-540 \mathrm{fs}^{[4]}$ for the solvation process as ${ }^{[5-6]}$ : $\mathrm{e}_{\text {quasi-free }}^{-} \rightarrow \mathrm{e}_{\text {wet }}^{-} \rightarrow \mathrm{e}_{\text {solvation }}^{-}$

In this process, the e-wet generation time is $300 \mathrm{fs}$, and the life time is 540fs. In the meantime, at lest $50 \%$ e-quasi-free is turned into e-wet. Computational simulation has shown that the structure of the hydrated electron is with approximately six water molecules with their $\mathrm{OH}$ bonds directed toward the electron. This is called the Kevan geometry ${ }^{[7]}$. Hence, a solvated electron looks similar to an ordinary solvated anion. In the solvation process, the free energy of the electron is reduced ${ }^{[8]}$, and the distance of the solvated electron can travel in water is less than $0.89 \mathrm{~nm}^{[9]}$. Nevertheless, according to the literature the transport distance of the electron in some proteins and DNA can be as long as $3 \mathrm{~nm}$ to $40 \mathrm{~nm}$. The conventional explanation for this mechanism is the quantum mechanical electron tunneling ${ }^{[10]}$. However, electron salvation data showed that this tunneling process was prohibited since the free electron from the donor must first be solvated and became the hydration electron $\mathrm{e}_{\text {aq }}^{-}$before being transported. 
Thus, we believe that a kind of transport proteins must exist in the cellular environment which can assist the transport of the solvated electron $\mathrm{e}_{\mathrm{aq}}^{-}$. At the end of the transport process, the transport proteins can supply energy to the $e_{\text {aq }}^{-}$to enable it to release the free electron which then to be transferred to the electron acceptor. Thus, we now propose a detailed

pathway for the endocellular electron transfer as the following:

$\mathrm{e}_{\text {quasi-free }}^{-} \rightarrow \mathrm{e}_{\text {wet }}^{-} \rightarrow \mathrm{e}_{\text {solvation }}^{-} \rightarrow$ transport proteins $\rightarrow$ fill energy $\rightarrow$ $\mathrm{e}_{\text {quasi-free }}^{-} \rightarrow$ acceptor

Conclusions:

By analyzed the electron salvation in endocellular environment, we give a picture on the electron transfer in cell. We discover that the electron salvation process prohibited the electron freely transfer in cell since the free electron from the donor must first be solvated and became the hydration electron $\mathrm{e}_{\text {aq }}^{-}$before being transported. We set-up a new theoretical picture to describe the hole process of electron transfer in cell.

Acknowledgements: We thank $\operatorname{Dr}$ Ma Yun-Qi's (Lanzhou Institute of Chemical Physics) useful discussion.

Author Contributions: Dr Yan Liu wrote the paper, he was the only one author in this paper. 
Additional Information: The author has no financial interests on this paper.

References:

[1] Giese B. Electron transfer in DNA Current Opinion in Chemical Biology (1998) , $6,235-243$.

[2] Shap R E., Chapman S. K. Mechanism for regulating electron transfer in multi-center redox proteins Biochimica et Biophysica Acta (1999) , $1432, \quad 143-158$

[3] Hart E. J., Boag J. W. Absorption spectrum of the hydrated electron in water and in aqueous solution J. Am. Chem. Soc. (1962), 84, 4090-4095

[4] Assel M., Laenen R., Laubereau A. Femtoescond sovation dynamics of solvated electrons in neat water Chemical Physics Letters (2000) , 317 , $13-22$

[5] Lu H., Long F. H., Bowman R. M. Femtosecond studies of electron-cation geminate recombination in water J. Phys. Chem. (1989) , 9, $327-28$

[6] Shi X., Long F. H., Lu H. Femtosecond electron solvation kenetics in water J. Phys. Chem. (1996)， 100，11903-11906

[7] Kevan L. Solvated electron structure in glassy matrices Acc. Chem. Res. (1981), 14, 138-145

[8] Han P., Bartels D. M. Reevaluation of Arrhenius parameters for $\mathrm{H}+\mathrm{OH}$ 
$\rightarrow\left(e_{\text {aq }}^{-}\right)+\mathrm{H}_{2} \mathrm{O}$ and the enthalpy and entropy of hydrated electron J. Phys. Chem. (1990) , 96, 4899-4906

[9] Schmidt K. H., Han P., Bartels D. M. Radiolytic yields of the hydrated electron from transient conductivity: Improved calculation of the hydrated electron coefficient and analysis of some diffusion-limited reaction rates J. Phys. Chem. (1995) ， 99， 10530-10539 [10] Beratan D. N., Skourtis S. S. Electron transfer mechanisms Current Opinion in Chemical Biology (2002), 2, 235-243 